Marquette University

e-Publications@Marquette

Biomedical Sciences Faculty Research and

Publications

Biomedical Sciences, Department of

$10-14-2020$

\title{
Marking Time: Colorful New Insights into Master Clock Circuits
}

Deborah A.M. Joye

Jennifer A. Evans

Follow this and additional works at: https://epublications.marquette.edu/biomedsci_fac

Part of the Neurosciences Commons 
Marquette University

e-Publications@Marquette

\section{Biomedical Sciences Faculty Research and Publications/College of Health Sciences}

This paper is NOT THE PUBLISHED VERSION.

Access the published version via the link in the citation below.

Neuron, Vol. 108, No. 1 (October 14, 2020) : 2-5. DOI. This article is (C) Elsevier (Cell Press) and permission has been granted for this version to appear in e-Publications@Marquette. Elsevier (Cell Press) does not grant permission for this article to be further copied/distributed or hosted elsewhere without the express permission from Elsevier (Cell Press).

\section{Marking Time: Colorful New Insights into Master Clock Circuits}

Deborah A.M. Joye

Department of Biomedical Sciences, Marquette University, Schroeder Complex, Milwaukee, WI Jennifer A. Evans

Department of Biomedical Sciences, Marquette University, Schroeder Complex, Milwaukee, WI

A neural clock controls what we do each day, and understanding its circuitry is important for health. In this issue of Neuron, Shan et al. visualize molecular rhythms in subtypes of master clock neurons to test principles of cell identity and network wiring.

Knowing the time of day is important, and not just so you can attend your next Zoom meeting on time. Over the years, clocks have been built to be more reliable, precise, robust to perturbation, locally tunable, and globally coordinated. And yet, our bodies have provided their own solution. Daily rhythms in biology are pervasive, regulating everything from cell division to cognitive function. Many daily rhythms persist without external time cues, with a period that approximates $24 \mathrm{~h}$ (i.e., circadian), reflecting programming by an endogenous clock. The gears of this clock are genetic, driven by transcriptional-translational feedback loops that regulate protein expression, and function across the day-night cycle (Buhr and Takahashi, 2013). At its core, this mechanism involves transcriptional activation by CLOCK::BMAL1, with later feedback repression from the protein products PERIOD and CRYPTOCHROME. This genetic control mechanism operates in nearly every cell of the body and 
regulates more than $40 \%$ of the genome (Zhang et al., 2014), thus requiring precise organization at both the intra- and intercellular levels. The master clock of this system is the suprachiasmatic nucleus (SCN) of the anterior hypothalamus (Antle et al., 2003; Evans, 2016; Hastings et al., 2018). The SCN is necessary for daily timekeeping because it ensures system-level coordination and serves as a critical interface between the body and the environment due to its direct connection with the retina.

From its first discovery, circadian biologists have wondered how the SCN is able to produce overt rhythms that are precise, robust, and yet adaptable. Local SCN circuits (1) bolster intracellular timekeeping, (2) maintain intercellular synchrony, and (3) encode environmental conditions to promote optimal health in a changing world. These emergent properties are rarely seen in other clock tissues. Which signals are critical for intercellular coupling in the SCN network? Is it organized like a democracy or a dictatorship? Is the intrinsic clock of a specific SCN cell type absolutely critical to the function of the whole network? Previous work has shown that different types of SCN cells have specialized roles and that peptide signaling sustains SCN function at the network level (Antle et al., 2003; Evans, 2016; Hastings et al., 2018). However, SCN circuits are still not well understood.

SCN neurons can communicate using GABA and more than 25 different peptides. Of these many different signals, one neuropeptide is best understood. Vasoactive intestinal polypeptide (VIP) is expressed in SCN neurons that process retinal inputs and regulate other $\mathrm{SCN}$ neurons, including those that express arginine vasopressin (AVP). Both VIP+ and AVP+ neurons provide outputs to downstream tissues, and both neuropeptides can act locally to modulate SCN function (Antle et al., 2003; Evans, 2016; Hastings et al., 2018). Germline loss of VIP or its cognate receptor (VPAC2) disrupts overt rhythms, alters behavioral responses to light, compromises SCN cellular rhythms, and leads to loss of SCN synchrony (Antle et al., 2003; Evans, 2016; Hastings et al., 2018). In short, the SCN seems to need VIP. However, it is also evident that other neuropeptides can modulate SCN function. For example, GABA and AVP-mediated V1 receptor signaling also act locally (Evans, 2016; Hastings et al., 2018), and different SCN neurons may operate in a context-specific manner (Smyllie et al., 2016). Thus, questions remain concerning the roles and rules by which different classes of SCN neurons operate, how this heterogeneous population coordinates, and which signals are critical.

To address these questions, Shan et al. (2020) have developed a novel mouse model that allows one to visualize genetic timekeeping in specific cells. Real-time imaging with optical reporters has long been used to visualize cellular timekeeping in a range of organisms (Welsh et al., 2005). Members of this research team engineered one of the best mammalian models for tracking genetic timekeeping-the PERIOD2::LUCIFERASE knockin mouse (reviewed in Welsh et al., 2005). In this mouse model, firefly luciferase produces a bioluminescent signal that reports the state of the critical clock gear PERIOD2. In the past, this type of approach has been used to track different clock genes in the same tissue, and it has been coupled with fluorescence to monitor different intracellular rhythms (e.g., Ca ${ }^{2+}$ rhythms) in SCN neurons versus glia (Hastings et al., 2018). However, no study to date has been able to monitor genetic timekeeping in defined subtypes of SCN neurons. Here, the authors devise an elegant approach by developing a Cre-dependent color-switching luciferase model. In this mouse, cells not only produce bioluminescence when PERIOD2 is expressed, but the type of neuron is indicated by the color of that light (green in the presence of Cre versus red in its absence). The authors have also developed advanced imaging systems that discriminate each bioluminescent signal, allowing comparison of rhythms in the Crelabeled neurons versus other neurons in the network.

So now that we can color-code timekeeping in specific neurons, do different types of SCN neurons have distinct clock properties? The authors first examine cellular rhythms in SCN slices ex vivo, using available Cre- models to switch the color of luciferase specifically in VIP or AVP neurons. The authors find that VIP neurons have a slower clock relative to the non-VIP neurons, and AVP neurons are faster than non-AVP neurons. Differences in clock speed were reflected in phasing of peak activity, as expected (Evans, 2016; Hastings et al., 2018). Further, the 
conclusion that VIP and AVP neurons differ in their clock properties is consistent with previous work investigating regional rhythms and AVP/VIP release ex vivo (Antle et al., 2003; Evans, 2016; Hastings et al., 2018). Perhaps more surprising, cellular phases in green-labeled neurons were not less variable than in red neurons, despite the former containing only one neurochemical class. Thus, each Cre+ subgroup is highly heterogeneous in phase, raising the possibility that there are subclusters within each SCN class. These findings also prompt the question of whether the properties measured in this slice preparation are intrinsic to each cell type or driven by network signaling.

To probe how network signaling contributes under these conditions, the authors next used tetrodotoxin (TTX) to inhibit action potentials by interfering with voltage-gated sodium channels. As expected, TTX caused intercellular desynchrony, which recovered upon TTX washout. TTX desynchronized all neuron types, indicating an important role for classic synaptic signaling. And yet, TTX disrupted phase coherence among VIP neurons more than any other type. This observation raises the possibility that SCN neurons use different modes of communication, some of which are more resistant to TTX. Indeed, previous work has demonstrated that paracrine neuropeptide signaling can restore SCN synchrony and that SCN neurons exhibit dense-core vesicle release from dendrites (Evans, 2016; Hastings et al., 2018). Thus, this suggests the exciting possibility that some SCN neurons may be more resilient to the disruptive effects of TTX because they are coupled by non-synaptic communication.

Next, the authors asked whether the molecular clock is required in AVP and VIP neurons by also using Cre to knockout Bmal1. Deletion of Bmal1 is a single-gene technique for "breaking" the molecular clock, making it an exceptional tool for testing whether it is necessary in specific cell types. The authors demonstrate that loss of the molecular clock in AVP neurons results in greater SCN desynchrony during TTX, which does not fully recover after washout. In contrast, absence of the VIP clock does not increase the effect of TTX, and the network is able to recover as normal. To further explore network communication with and without each neuron-specific clock, the authors next developed a new index of synchrony-the maximal information coefficient (MIC), which quantifies the strength of correspondence between rhythms in different cell types. Interestingly, the MIC finds that even before TTX, coupling is weaker without an AVP neuron clock. Application of TTX further weakens this coupling, and it remains weak among all neuron pairs even after washout. Collectively, these results suggest that the molecular clock in AVP neurons is necessary for normal SCN function (Figure 1). Moreover, these data suggest that the molecular clock in AVP neurons controls their ability to communicate even when action potentials are inhibited, possibly via non-synaptic modes of communication (Figure 1). Further investigation of how loss of Bmal1 affects cellular physiology in AVP and other SCN neurons may provide additional insight into how the AVP clock increases network resiliency under these conditions. 


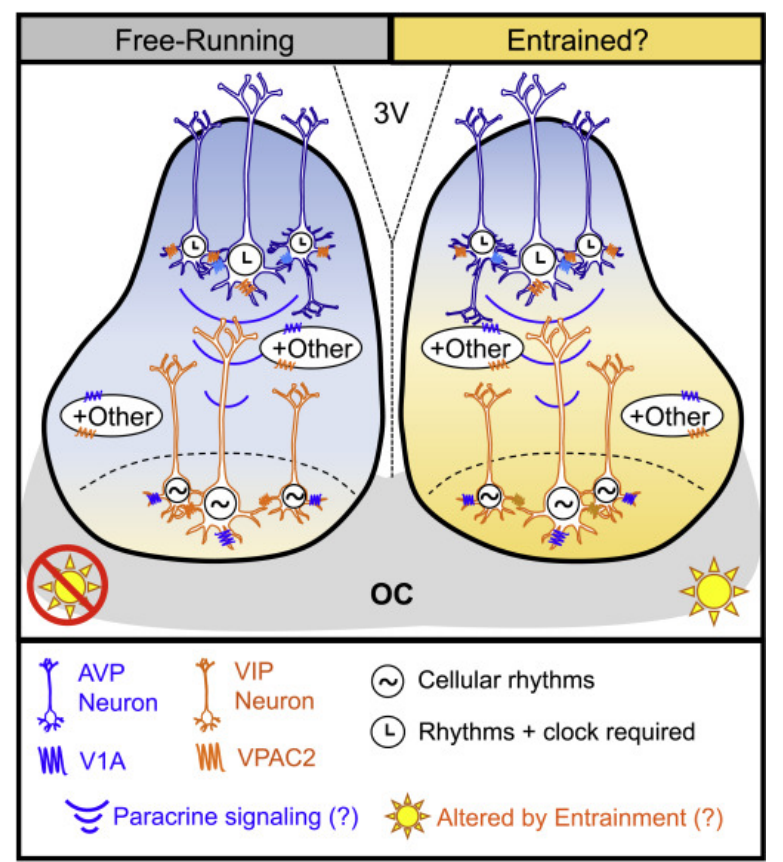

Figure 1. Shan et al. (2020) Demonstrate that the SCN Clock Contains Cellular Gears that Differentially Require Their Molecular Clock for Network Function

Left: the AVP neuron molecular clock is required for SCN communication in vitro. Under free-running conditions, cellular interactions maintain SCN synchrony, TTX interferes with this coupling, and recovery requires the molecular clock in AVP neurons. In contrast, the molecular clock in VIP neurons appears unnecessary under these conditions despite strong cellular rhythms displayed by this neuronal class. Right: SCN circuits may differ in vivo and may change with photic input. It remains possible that the VIP molecular clock also contributes to SCN network function, albeit under different conditions.

$3 \mathrm{~V}$, third ventricle; OC, optic chiasm. Note: gradient depicts potential for different SCN circuitry in place under free-running versus entrained conditions.

Having found that loss of the AVP neuron clock causes SCN deficits in vitro, the authors next tested how this would influence daily rhythms in vivo. Interestingly, all mice entrained normally, and free-running behavior in constant darkness was unaffected initially, suggesting that the circadian system is very resilient to loss of the AVP neuron clock in vivo. However, in vitro deficits were most readily detected when synaptic signaling was disrupted with TTX, suggesting that a challenge to the system may be necessary. To disrupt SCN communication in vivo, the authors exposed mice to constant light, which desynchronizes SCN neurons (Evans, 2016; Hastings et al., 2018). Consistent with in vitro findings, constant light caused behavioral after-effects specifically in mice lacking an AVP neuron clock. But another striking feature of these data is that aftereffects in AVP-clockless mice disappeared when the system was re-entrained to a normal photoperiod. Although it is not clear how this may occur, this suggests that the system can be "fixed" even in the absence of an AVP neuron clock. In contrast, VIP-clockless mice did not respond differently from wild-type, suggesting that loss of the VIP neuron clock does not compromise the ability to entrain normally or cope with constant light. This is somewhat surprising, given that VIP neurons and VIP itself are involved critically in photic entrainment (Mazuski et al., 2018; also Evans, 2016; Hastings et al., 2018). Thus, important questions remain regarding how the molecular clock and other factors interact to regulate cellular rhythms and SCN neuropeptide expression.

Lastly, to gain insight into how the neuron-specific clock affects intercellular signaling, the authors analyzed AVP, VIP, V1A, and VPAC2 levels in the SCN prior to the start of the active phase. All four of these proteins are rhythmically expressed in the SCN, and this time of day corresponds to peak AVP and VIP expression in wild-type mice under entrained conditions (Antle et al., 2003; Evans, 2016; Hastings et al., 2018). The authors found that 
AVP and V1A are reduced in mice lacking an AVP or VIP clock but that VPAC2 is unaffected. This latter finding prompts the question of which factors might drive VPAC2 rhythms (Evans, 2016; Hastings et al., 2018). Because many different types of SCN cells express VPAC2, it remains possible that its expression does depend on molecular clock function in a subset of cells, or it may be that changes in VPAC2 are only evident at other phases of the circadian cycle. Thus, it would be useful to build on these data to investigate how neuron-specific loss of the molecular clock alters neuropeptide and receptor expression over the circadian cycle. Lastly, the authors found that VIP expression was elevated in AVP-clockless mice, providing the first clear evidence that AVP neurons regulate VIP despite there being very few synaptic projections in this direction (Antle et al., 2003; Evans, 2016; Hastings et al., 2018). It is possible that AVP neurons could communicate with VIP neurons via non-synaptic signaling or via synaptic projections to other classes of SCN neurons (Figure 1).

Overall, these data provide new insight into how the cellular gears of the master clock fit together and highlight a number of exciting questions that will benefit from continued use of this new model. For instance, the AVP clock is important for SCN function, but how do AVP neurons influence other SCN cells? Is this synaptic and/or paracrine-mediated communication, and which specific receptor mechanisms transduce this signal to alter the molecular clock in target cells? If VIP neurons do not need a molecular clock, why and how do these neurons display such strong cellular rhythms? SCN function was fairly robust to loss of both the AVP and VIP neuron clocks, raising the possibility that other types of SCN cells may contribute. Lastly, are SCN circuits wired differently across the lifespan, according to biological sex, and/or by the environment? Do the rules and principles revealed here apply equally to other forms of SCN coupling? Perhaps intact clock function in VIP neurons is important but only becomes required under specific conditions and/or modulates other properties not examined here. Indeed, SCN organization and signaling can vary with environmental experience (Evans, 2016; Hastings et al., 2018), so perhaps there is more than one wiring diagram from which the SCN network can operate. Precedent for clock circuit flexibility stems from work in Drosophila showing that different cells can control circadian output under different conditions (Stoleru et al., 2004). Perhaps it is also true that the SCN network is designed not as a pure democracy or dictatorship, but instead as a hierarchical (and flexible) system of specialized committees. Perhaps this circuit model uniquely provides the precision, robustness, and adaptability required by our predictable and yet changing world.

\section{Acknowledgments}

The authors are supported by funding from the NIH (R01NS091234) and the Whitehall Foundation (2014-12-65).

\section{References}

Antle et al., 2003 M.C. Antle, D.K. Foley, N.C. Foley, R. Silver. Gates and oscillators: a network model of the brain clock. J. Biol. Rhythms, 18 (2003), pp. 339-350

Buhr and Takahashi, 2013 E.D. Buhr, J.S. Takahashi. Molecular components of the Mammalian circadian clock. Handb. Exp. Pharmacol., 217 (2013), pp. 3-27

Evans, 2016 J.A. Evans. Collective timekeeping among cells of the master circadian clock. J. Endocrinol., 230 (2016), pp. R27-R49

Hastings et al., 2018 M.H. Hastings, E.S. Maywood, M. Brancaccio. Generation of circadian rhythms in the suprachiasmatic nucleus. Nat. Rev. Neurosci., 19 (2018), pp. 453-469

Mazuski et al., 2018

C. Mazuski, J.H. Abel, S.P. Chen, T.O. Hermanstyne, J.R. Jones, T. Simon, F.J. Doyle 3rd, E.D. Herzog. Entrainment of circadian rhythms depends on firing rates and neuropeptide release of VIP SCN neurons. Neuron, 99 (2018), pp. 555-563

Shan et al., 2020

Y. Shan, J.H. Abel, Y. Li, M. Izumo, K.H. Cox, B. Jeong, S.H. Yoo, D.P. Olson, F.J. Doyle 3rd, J.S. Takahashi, 
Dual-color single-cell imaging of the suprachiasmatic nucleus reveals a circadian role in network synchrony. Neuron, 108 (2020), pp. 164-179

Smyllie et al., 2016 N.J. Smyllie, J.E. Chesham, R. Hamnett, E.S. Maywood, M.H. Hastings. Temporally chimeric mice reveal flexibility of circadian period-setting in the suprachiasmatic nucleus. Proc. Natl. Acad. Sci. USA, 113 (2016), pp. 3657-3662

Stoleru et al., 2004 D. Stoleru, Y. Peng, J. Agosto, M. Rosbash. Coupled oscillators control morning and evening locomotor behaviour of Drosophila. Nature, 431 (2004), pp. 862-868

Welsh et al., 2005 D.K. Welsh, T. Imaizumi, S.A. Kay. Real-time reporting of circadian-regulated gene expression by luciferase imaging in plants and mammalian cells. Methods Enzymol., 393 (2005), pp. 269-288

Zhang et al., 2014 R. Zhang, N.F. Lahens, H.I. Ballance, M.E. Hughes, J.B. Hogenesch. A circadian gene expression atlas in mammals: implications for biology and medicine. Proc. Natl. Acad. Sci. USA, 111 (2014), pp. $16219-16224$ 